\title{
Initiation of Femtosecond Laser Machined Ripples in Steel Observed by Scanning Helium Ion Microscopy (SHIM)
}

\author{
Bert Huis in 't Veld ${ }^{* 1,2}$ and Hans van der Veer ${ }^{* 2}$ \\ ${ }^{* 1}$ University of Twente, Faculty of Engineering Technology, Applied Laser Technology, Driener- \\ lolaan 5, Enschede, Post Office Box 217, 7500 AE Enschede, The Netherlands \\ E-mail: a.j.huisintveld@utwente.nl \\ *2 TNO Science and Industry, Department Materials Technology, De Rondom 1, Eindhoven, \\ Post Office Box 6235, 5600 HE Eindhoven, The Netherlands
}

\begin{abstract}
The physics of ablation has been studied experimentally with ultrashort laser pulses of $210 \mathrm{fs}$ with a wavelength of $800 \mathrm{~nm}$. A specimen of highly alloyed steel (alloy $800 \mathrm{H}$ ) has been used. After the laser ablation experiments the surface has been inspected using SEM, AFM and scanning helium ion microscopy (SHIM). At the lowest fluence spherical bubbles are observed consisting of a mixture of gas and liquid having diameters in the range 20-90 $\mathrm{nm}$. With increasing fluence the chain of events that has been observed consists of bubble forming and initiation of pre-ripples followed by a transformation towards regular course ripples with an orientation perpendicular to laser polarization direction. The pre-ripples have a different orientation that is not understood yet.

DOI: $10.2961 / \mathrm{j} 1 \mathrm{mn} .2010 .01 .0007$
\end{abstract}

Keywords: initiation, bubbles, ripples, pre-ripples, femtosecond, ultrashort laser pulses, helium microscopy, laser ablation

\section{Introduction}

Ripple forming on surfaces of metals and other materials is widely observed, starting already a few years after the invention of lasers in 1960. Although the physics of ripple forming are studied worldwide, the initiation and growth mechanism still contain unanswered questions.

The study presented here is a continuation of a previous study [1] where pre ripples were reported and AFM images were presented. These pre ripples have spacings or wavelengths in the range $100-200 \mathrm{~nm}$ and an amplitude of about $10 \mathrm{~nm}$. The orientation of pre ripples is far from perpendicular to the polarization direction of the laser light. It was observed that pre ripples transform into regular ripples with an orientation perpendicular to the polarization direction of the laser beam applied. The initiation started on small carbides present in the microstructure of the alloy used.

The wavelength of regular ripples depends on the fluence. Applying a laser spot with a Gaussian energy distribution for making a laser track on a metal surface, the largest wavelengths are found in the centre (typically $400 \mathrm{~nm}$ ) whereas the edges of a track contain ripples with smaller wavelengths (typically $250 \mathrm{~nm}$ ).

Models for explaining the initiation and growth of ripples can be divided into interference models [2] and models where self organizing is the main mechanism [3]. Pre ripples, varying ripple wavelengths and machine speed dependence are not consistent with the model of an interference modulated energy input transforming directly into the observed surface modulation. The model of self organizing structure formation can explain varying spacings, however the origin of pre ripples and the transition towards regular ripples are not explained.

To improve the understanding of ripple forming new experiments have been done on alloy $800 \mathrm{H}$, an iron based alloy with $30 \%$ nickel and $20 \%$ chromium. Low laser fluence levels have been applied to generate pre ripples in this alloy. Although this alloy contains high levels of nickel and chromium, much higher than the most common austenitic stainless steel, we have experienced that the interaction with short laser pulses of these iron based steel is similar.

To study the details of surface textures with pre ripples a relatively new method has been used for surface analysis: Scanning Helium Ion Microscopy (SHIM). With this technique the initiation of pre ripples and transformation towards regular ripples have been observed as present on the specimen surface after laser machining.

\section{1 fs laser system}

A titanium sapphire based laser system with a central wavelength of $800 \mathrm{~nm}$ was used for generation of the laser pulses. A seed/oscillator combination (Coherent Vitesse Duo) together with a regenerative amplifier (Coherent RegA9000) delivered pulses at $50 \mathrm{kHz}$ repetition rate with $2 \mathrm{~mW}$ average power. The pulse length, measured by a second order auto correlator, was adjusted to $210 \mathrm{fs}$ for all experiments. A combination of a rotary $\lambda / 2$ wave plate and a beam splitting polarizing cube served as attenuator. The beam is horizontally polarized.

Manipulation of the bundle over the sample was accomplished by a two mirror galvo scanner system (Scanlab Scangine 14). A $100 \mathrm{~mm}$ f-theta lens (fused silica) focused the beam to an elliptical spot size with a long size of about $25 \mu \mathrm{m}$ and a short size of about $17 \mu \mathrm{m}$. Due to the Gaussian energy distribution the effective spot size is smaller, here about $20 \mu \mathrm{m}$, see figure 5 .

The average fluence was determined by measuring the beam power at the exit of the scanner system with a power meter and dividing it by the repetition rate and the surface area of the laser spot. In all experiments the angle of inci- 
dence of laser light was perpendicular to the specimen surface.

\subsection{Specimen}

Alloy $800 \mathrm{H}$ is a highly alloyed (table 1) austenitic steel. The specimen used was cut from a piece of steel used for one year in industry at about $600{ }^{\circ} \mathrm{C}$. Due to the industrial exposure to $600{ }^{\circ} \mathrm{C}$ small intergranular carbides have grown. In the previous study it was shown that the transformation of pre ripples towards regular ripples initiated on these small intergranular carbides. In order to remove these initiation sites we decided to dissolve these carbides by a solution treatment for 2 hours at $1100{ }^{\circ} \mathrm{C}$ with a water quench.

The specimen has been slightly etched before laser machining during 3 minutes with so-called V2A beize, an acid etchant with $\mathrm{HCl}$ and $\mathrm{HNO}_{3}$. The microstructure of this alloy is depicted in figure 1 that shows the etched grain boundaries. Like many other austenitic steels this alloy contains twins, which are areas with straight boundaries and a structure that is mirror symmetrical from the surrounding grain.

Table 1 Chemical composition of X10NiCrAlTi 3220 (alloy $800 \mathrm{H}$ )

\begin{tabular}{ccccccccc}
\hline Element & $\mathrm{Fe}$ & $\mathrm{Ni}$ & $\mathrm{Cr}$ & $\mathrm{Ti}$ & $\mathrm{Al}$ & $\mathrm{Mn}$ & $\mathrm{Si}$ & $\mathrm{C}$ \\
\hline Wt $\%$ & bal & $30-$ & $19-$ & $0.15-$ & $0.15-$ & $\leq 2.0$ & $\leq 1.0$ & $\leq .12$ \\
& & 34 & 23 & 0.60 & 0.60 & & &
\end{tabular}

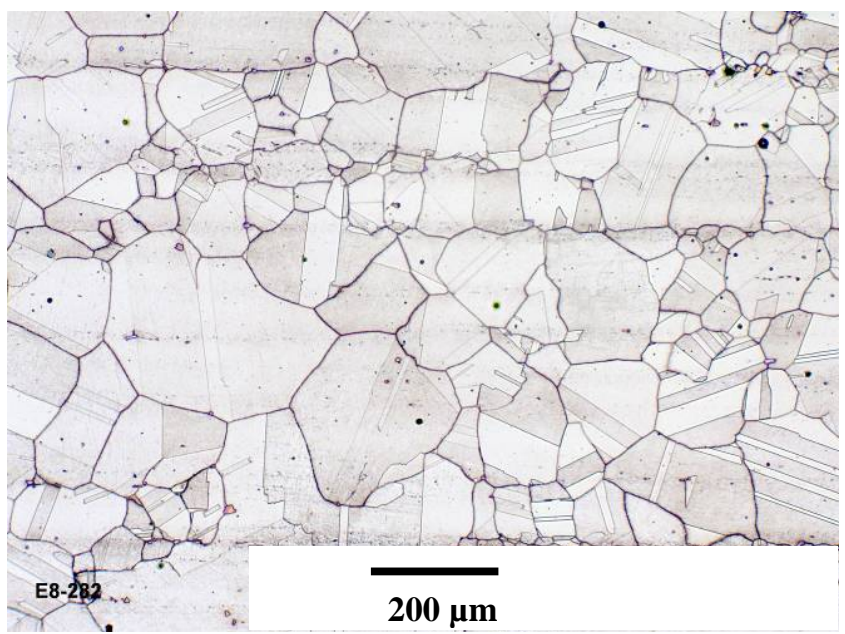

Fig. 1 Etched alloy $800 \mathrm{H}$ with a microstructure dominated by grain boundaries and twins (with straight boundaries)

\subsection{Surface analysis}

For surface analysis different techniques have been used: light microscopy, confocal light microscopy, scanning electron microscopy (SEM), atomic force microscopy (AFM) and scanning helium ion microscopy (SHIM) [4].

For an overview of well polished or etched surfaces light microscopy remains a powerful method. For better lateral resolution SEM is used with the advantage of a larger focus depth. For depth profiles and z-information in general, confocal light microscopy is used, however the lateral resolution of confocal light microscopy is limited. In this study pre ripples could not be imaged by confocal light microscopy. With AFM it is possible to image pre ripples and to measure the amplitude of this surface modulation [1]. SHIM has been used to study the nanometer sized details of laser machined surfaces, since $30 \mathrm{keV}$ helium ions generate secondary electrons in a very small image forming volume. Operation is basically analogous to a SEM except for using positive ions. The benefits of using helium ions for imaging are: ultrahigh resolution, different material contrast, good depth of focus and relatively low charging of insulators.

\section{Experimental approach}

Applying a fixed laser power of $2 \mathrm{~mW}$ the scan speed and number of overscans are varied as depicted in figure 2. The scan speed is increased from left to right. Starting at 50 $\mathrm{mm} / \mathrm{s}$ in the first column on the left to $100,200,400$ and in the right column $800 \mathrm{~mm} / \mathrm{s}$.

The laser tracks in the first row are made by a single scan. In the second row one overscan is applied. The laser tracks in the third row are made with five scans and the tracks in row 4 with 10 scans. The lowest row contains lines made with 20 scans.

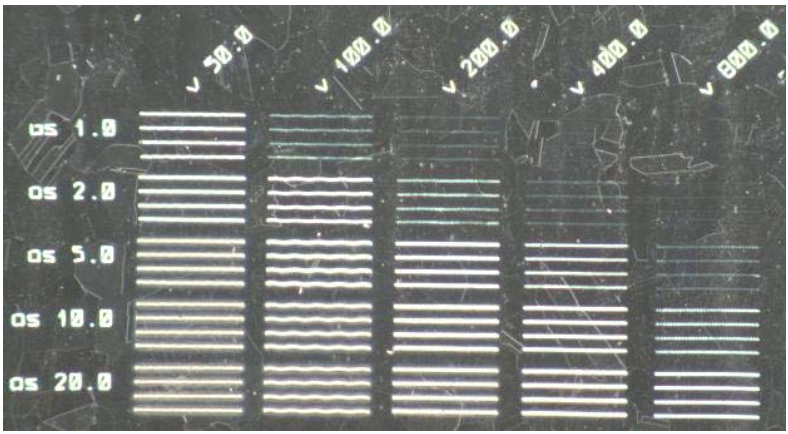

Fig. 2 Laser tracks on specimen surface. At each combination of scan speed and number of scans four identical lines are written. A single line is about $0.75 \mathrm{~mm}$ long.

For each combination of scan speed and number of scans four identical lines are written on the specimen. The lines written at $100 \mathrm{~mm} / \mathrm{s}$ are not straight; a vertical vibration is superimposed to the horizontal lines. This vibration is caused by the dynamic behavior of the scanner.

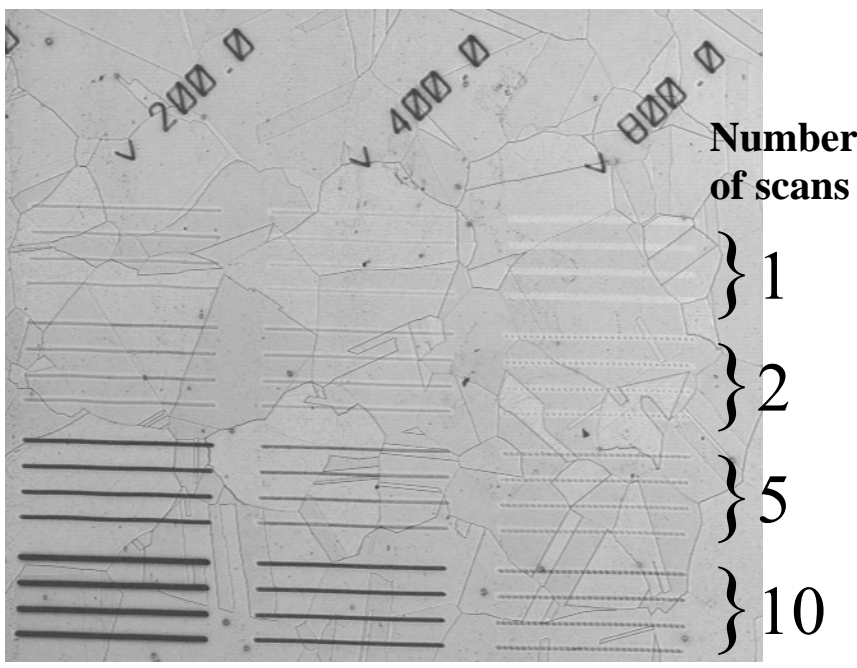

Fig. 3 Part of area depicted in Figure 2. Scan speed from left to right is 200,400 and $800 \mathrm{~mm} / \mathrm{s}$. The number of scans is $1,2,5$ and 10 . 
The four lines upper right in figure 2 are not visible. Therefore a second image is made, figure 3 . In figure 3 upper right, the four lines made with a single scan and a speed of $800 \mathrm{~mm} / \mathrm{s}$ are visible as light (white) lines. First impression is that the surface in the laser tracks has been cleaned. These lines have been analyzed further with SHIM.

Due to the etching procedure followed, the specimen contains steps with a typical step height of $100 \mathrm{~nm}$. This is imaged in figure 4 where two laser tracks made in two scans and a scan speed of $100 \mathrm{~mm} / \mathrm{s}$ are depicted.

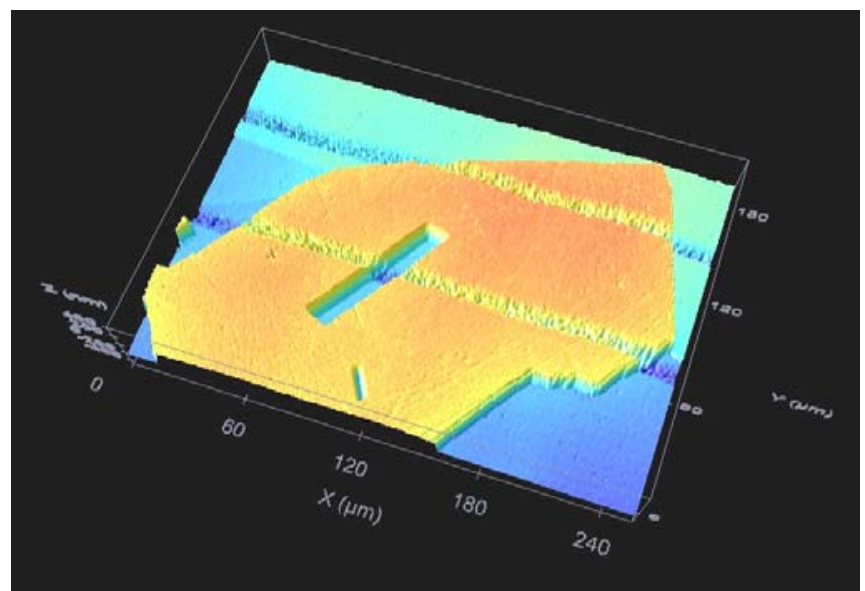

Fig.4 Confocal image of etched specimen surface containing steps. Two laser tracks are depicted. The length of the coloured area is $240 \mu \mathrm{m}$.

An average power of $2 \mathrm{~mW}$ and a repetition rate of 50 $\mathrm{kHz}$ implies that each pulse contains $40 \mathrm{~nJ}$ energy. Assuming a circular spot size with diameter $20 \mu \mathrm{m}$ an average energy intensity of $61 \mathrm{GW}$ per $\mathrm{cm}^{2}$ is calculated. Due to the Gaussian energy distribution the energy intensity in the central part of the laser spot will be about $122 \mathrm{GW}$ per $\mathrm{cm}^{2}$.

\section{Results}

\subsection{Lowest fluence}

The lines produced with the lowest fluence $\left(13 \mathrm{~mJ} / \mathrm{cm}^{2}\right.$ on average) are the upper right ones in figures 2 and 3. Due to the velocity of $800 \mathrm{~mm} / \mathrm{s}$ the laser beam shifts $16 \mu \mathrm{m}$ between two pulses. As a result adjacent pulses show some overlap, but the central parts of the laser spot do not overlap. This is depicted in Figure 5.

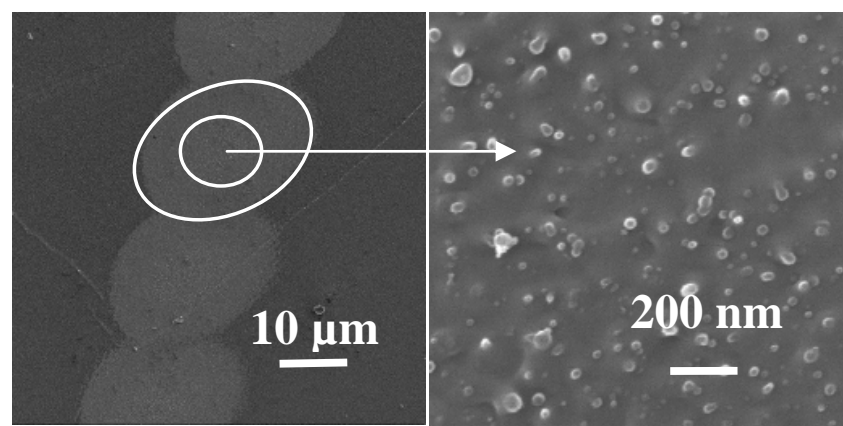

Fig.5 SHIM image of nano bubbles with diameters $20-50 \mathrm{~nm}$ in central part of an elliptical laser spot, the energy intensity was about $122 \mathrm{GW}$ per $\mathrm{cm}^{2}$ and small bubbles are formed.

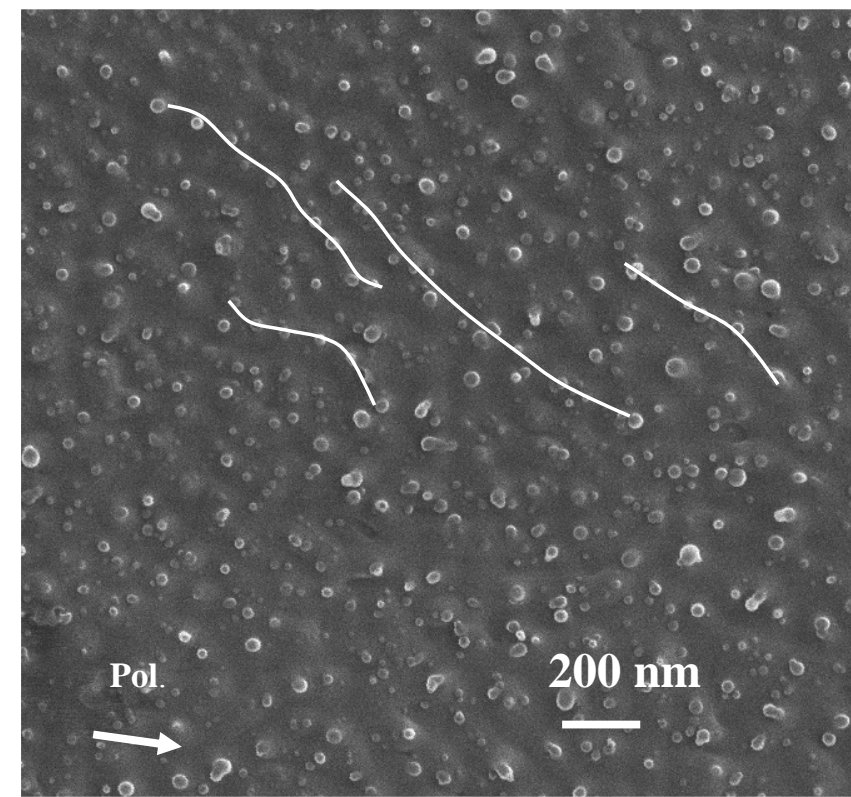

Fig. 6 Nano bubbles with diameters in the range $20-50 \mathrm{~nm}$. The surface has a certain waviness as can be concluded from the contrast variations. Bubbles are preferentially found on surface ridges. In the upper half of the image four lines are drawn connecting bubbles on a ridge.

Nucleation of gas bubbles and development of a heterogeneous phase of liquid and gas is already described in 1998 [5]. Phase explosion as a decomposition of a thermodynamically metastable homogeneous liquid into a mixture of liquid droplets and gas follow from molecular dynamics simulations [6]. Images of initiation and growing nano sized bubbles have not been made before. That bubbles show a tendency to initiate on surface ridges is indicated by a few white lines drawn in figure 6 .

\subsection{Effect of a second scan}

In the right column of figures 2 and 3 the second set of four lines is obtained by a second scan some $12 \mathrm{~ms}$ after the first one. After a second scan bubbles and pre ripples are found by SHIM in the central area of a laser pulse, figure 7. Bubbles are larger than those after the first scan in figures 5 and 6 and they are preferentially found on top of pre ripples. Obviously the ridges as found after the first scan (see figure 6) have been transformed into pre ripples.
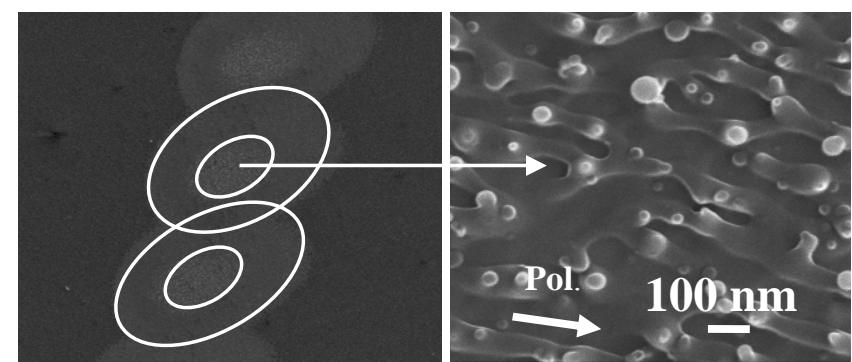

Fig.7 SHIM image of nano bubbles on pre ripples. Bubbles have diameters in the range $20-70 \mathrm{~nm}$, some are necked. Pre ripples have wavelengths of about $122 \mathrm{~nm}$.

These pre ripples have the same orientation as the surface ridges in figure 6 which is roughly the polarization direction of the laser beam as indicated in figure 7 . 


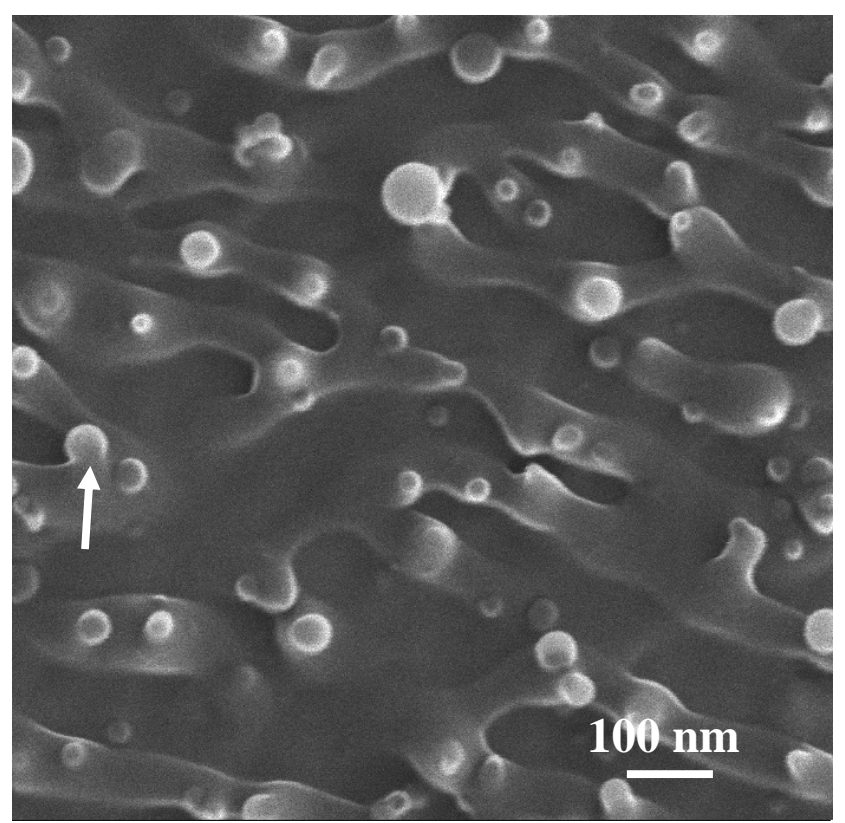

Fig. 8 Since the initiation of bubbles connected to pre ripples form the main result in this study, the image of fig 7 right, is printed a second time. Here the necking of one bubble (arrow) is well visible. By AFM the amplitude of pre ripples has been measured and a value of $32 \mathrm{~nm}$ (peak to valley) was measured.

\subsection{Effect of multiple scans}

With each scan more energy is pumped in the surface and also because the position of pulses in sequential scans is not exactly the same, the areas with pre ripples within a track are growing. After 5 scans within a track the individual laser spots still can be clearly recognized, after 10 scans the centre of the whole track is occupied with pre ripples and the first signs of regular ripples [7] appear, see figure 10.

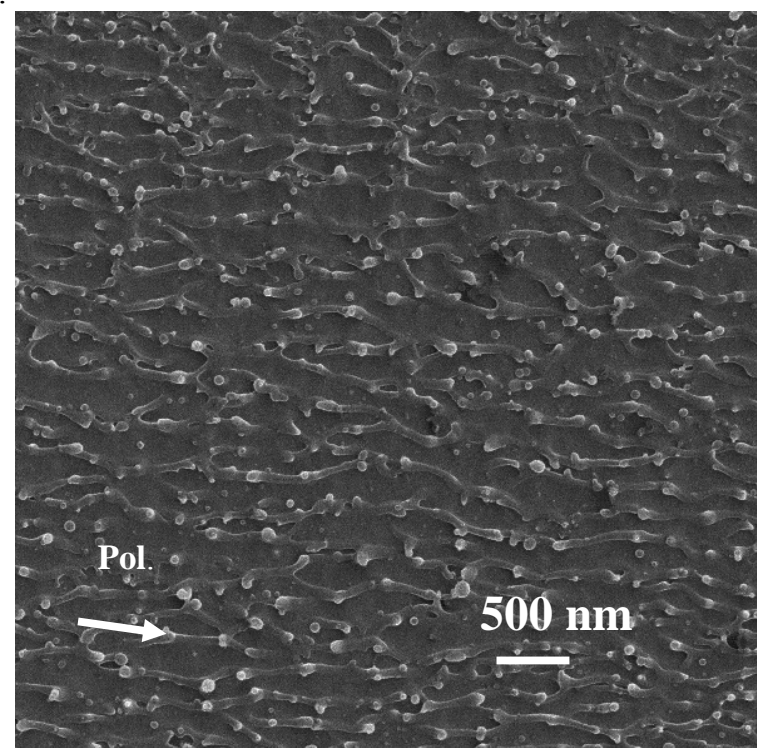

Fig. 9 SHIM image of a laser track after five scans (scan speed $800 \mathrm{~mm} / \mathrm{s}$ ). Area with bubbles and pre ripples. Almost all bubbles are connected to pre ripples; the wavelength of the pre ripples here is $172 \mathrm{~nm}$. Regular ripples are not found.

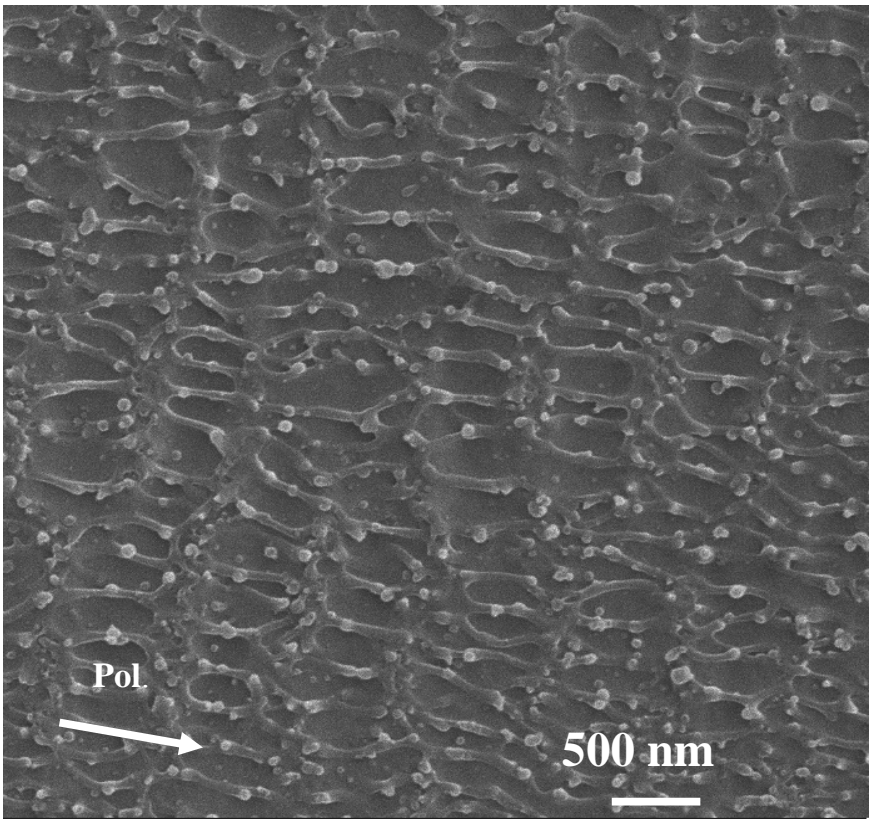

Fig. 10 SHIM image after 10 scans. Pre ripples have a wavelength of $192 \mathrm{~nm}$. Besides bubbles and pre ripples also the orientation of regular ripples can be clearly recognized.

Regular ripples with an orientation perpendicular to the polarization direction (figure 10) form apparently grooves in the surface under pre ripples. Pre ripples bridge the course regular ripples and there is a strong interaction between bubbles and pre ripples as can be seen in figure 11 .

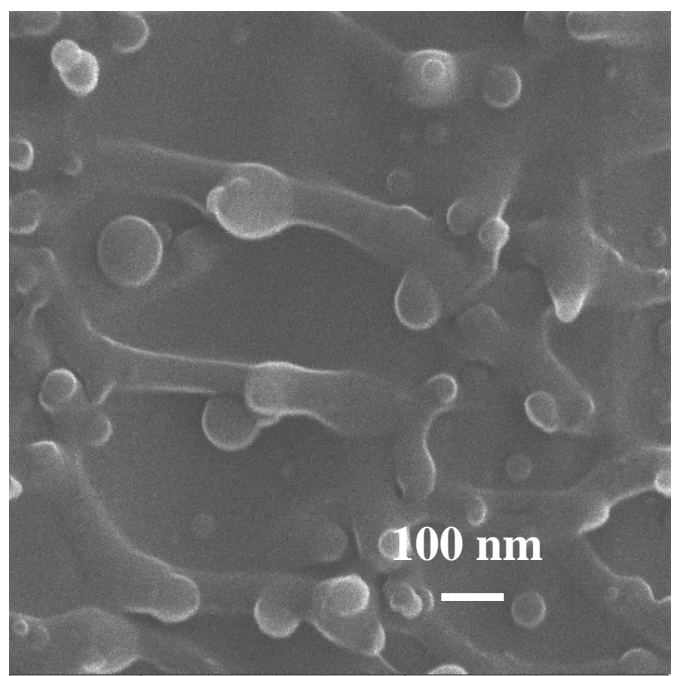

Fig. 11 SHIM image after 10 scans at $800 \mathrm{~mm} / \mathrm{s}$. Bubble has become a part of pre ripple. The diameter of the largest bubble is about $110 \mathrm{~nm}$.

After 20 scans the regular ripples are the dominant surface feature. Pre ripples and bubbles can still be observed. As can be seen in figure 12, regular rinnles are larger than pre ripples. The wavelength of the regular ripples in figure 12 is about $500 \mathrm{~nm}$. 


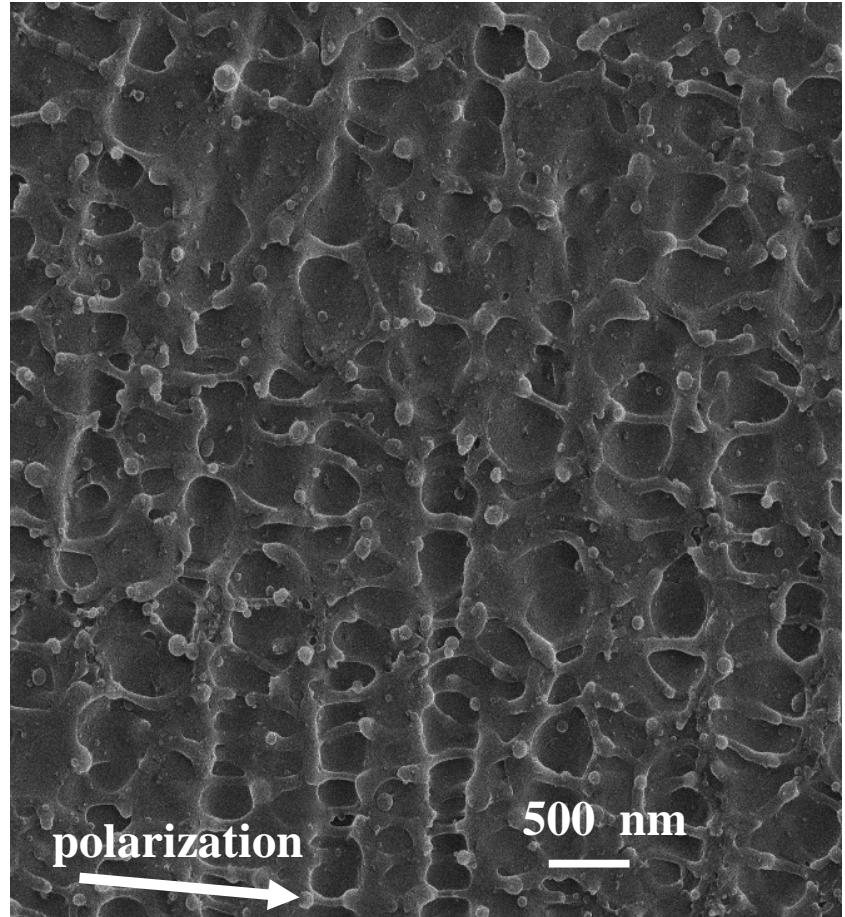

Fig. 12 SHIM image of regular ripples formed after $\angle U$ scans at the highest speed of $800 \mathrm{~mm} / \mathrm{s}$.

The surface features in figure 12 can be interpreted as a ductile response to tensile stresses. The origin of tensile stresses can be found in thermal expansion of the surface layer due to heating and superfast cooling under influence of laser energy. Both pre ripples and regular ripples have a fixed, but different orientation with respect to the polarization of the laser beam. However, the surface effects (bubble forming and ripple forming) can only start in the nanosecond regime and need the time between two pulses $(20 \mu \mathrm{s})$ to develop.

The polarization of the laser beam is only present at the surface during the pulse, which is during $210 \mathrm{fs}$. So the surface remembers the polarization of the laser light used (long) after the end of the laser pulse. So the question is raised: What is the memory mechanism active at the surface? [8] A mechanism where electrical charges are distributed in the surface under influence of polarization are a possible mechanism and are possibly a part of the answer, but this needs further analysis.

\subsection{Influence of scan speed}

Going back to the top row of lines in figure 2 we have laser tracks formed with different scan speeds and all other parameters identical. Starting with a scan speed of $50 \mathrm{~mm} / \mathrm{s}$ figure 13 , we have been working with strongly overlapping pulses. The pulse diameter is about $20 \mu \mathrm{m}$ and the laser beam shifts $1 \mu \mathrm{m}$ per pulse, so we generate 20 overlapping pulses. The results are imaged in figure 14 . In figure $14 \mathrm{a}$

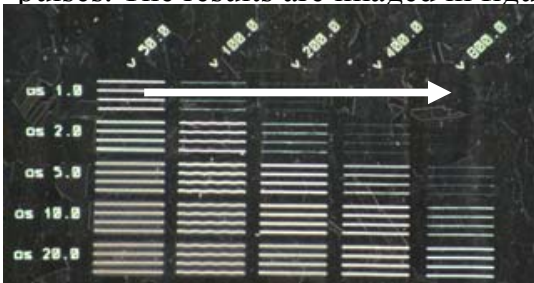

Fig.13 Lines made in one scan with increasing scan speed from left to right 50,100, 200, 400 and $800 \mathrm{~mm} / \mathrm{s}$

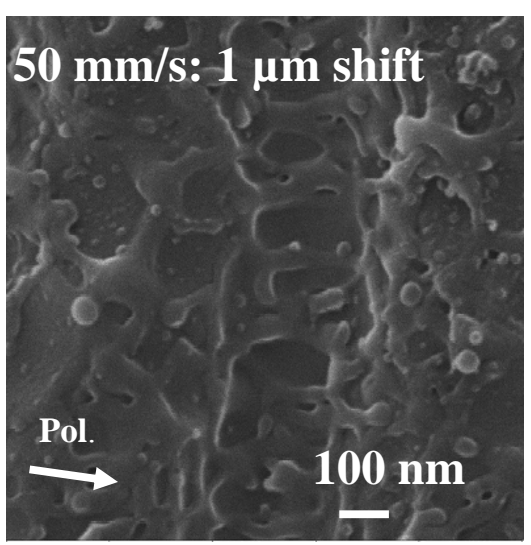

Fig. 14a

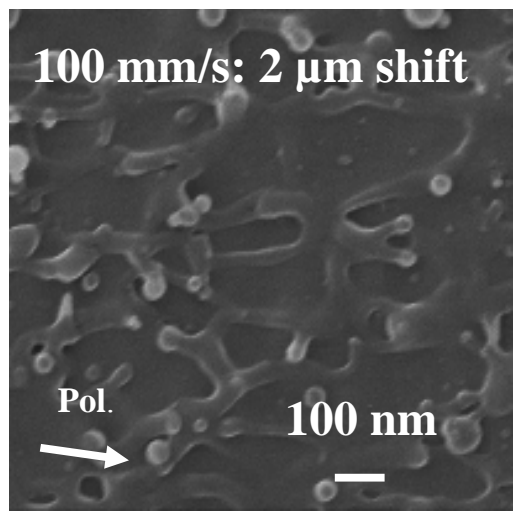

Fig. 14b

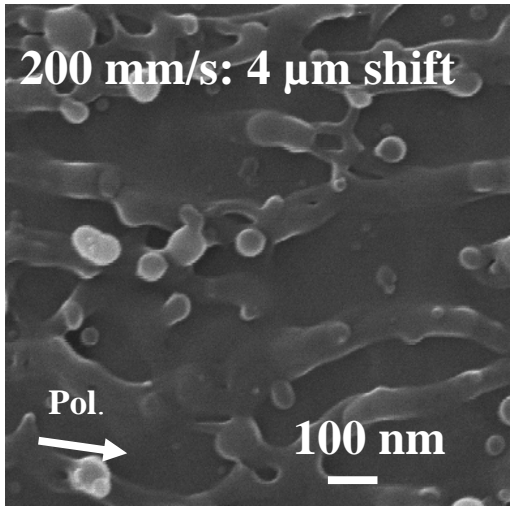

Fig. 14c

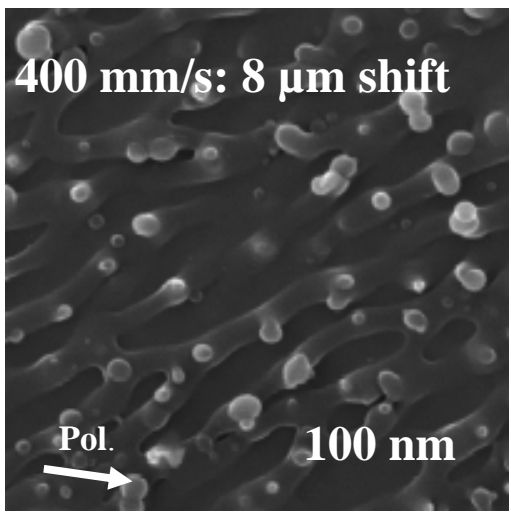

Fig. 14d

Fig.14 SHIM images after one scan. Increasing speed gives a larger shift between two pulses and reduces the overlap of sequential pulses. The degree of order increases from top to bottom.

The scan speed was $50 \mathrm{~mm} / \mathrm{s}$. In figure $14 \mathrm{~b}$ the scan speed was $100 \mathrm{~mm} / \mathrm{s}$ resulting in about 10 overlapping pulses. In 
figure $14 \mathrm{c}$ the scan speed was $200 \mathrm{~mm} / \mathrm{s}$ resulting in 5 overlapping pulses. In figure $14 \mathrm{~d}$ the scan speed was 400 $\mathrm{mm} / \mathrm{s}$ resulting in a shift between two pulses of $8 \mu \mathrm{m}$, with a pulse diameter of $20 \mu \mathrm{m}$, the overlap is 2.5 . Finally at the highest speed of $800 \mathrm{~mm} / \mathrm{s}$ the pulses show almost no overlap as can be seen in figure 5 and we obtained only bubbles with a certain waviness in the surface. As indicated before the average fluence of a single pulse is $13 \mathrm{~mJ} / \mathrm{cm}^{2}$ and this value will be higher in the centre due to the Gaussian energy distribution.

We observe that in figure $14 \mathrm{a}$ at the lowest speed a regular ripple is visible, whereas in figure $14 \mathrm{~d}$ a pre ripple with bubbles is visible. With increasing speed the overlap and the fluence is lowered resulting in more regular surface features. Comparing figures $14 \mathrm{~d}$ with figure 8 the influence of time between two pulses can be studied. In figure 8 the time between two pulses on same area is determined by the time between the first and second scan, which was a few ms. This is mainly waiting time before the second scan is started. In figure $14 \mathrm{~d}$ the time is controlled by the repetition rate of $50 \mathrm{kHz}$ and the time between two pulses is $20 \mu \mathrm{s}$.

The features in both figures are comparable. Both contain fine ripples connected with bubbles, although the width of ripples in figure $14 \mathrm{c}$ is a little smaller than in figure 8 . From this observation we conclude that the time between two pulses has an influence on the texture that is developing due to the laser energy input. The longer time (ms) between two pulses causes a coarser ripple size. Because the relative position of pulses in two scans is not exactly known, the energy input can play a role as well.

\subsection{Highest fluence}

The highest fluence is obtained at the lowest scan speed $(50 \mathrm{~mm} / \mathrm{s})$ and the highest number of scans (20), which are the conditions in the lower left corner in figure 2 . The fluence there is about $4.1 \mathrm{~J} / \mathrm{cm}^{2}$ and the width of the laser track is $19 \mu \mathrm{m}$, see figure 15 . In the centre of the track course regular ripples are observed, near the edges the regular ripples are finer. This has been observed before [1].

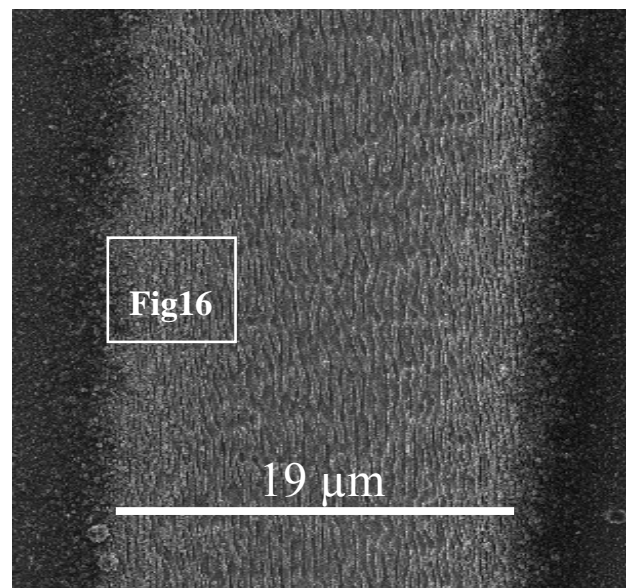

Fig.15 SHIM image of laser track made with 20 scans, $50 \mathrm{~mm} / \mathrm{s}$. Fluence $=0.5 \mathrm{~J} / \mathrm{cm}^{2}$.

and is a reflectance of the Gaussian energy distribution over the width of a laser track. The edge of the laser track in figure 15 is imaged again in figure 16. The edges contain some bubbles and large numbers of clustered particles. Outside the rippled area of $19 \mu \mathrm{m}$ width a large amount of clustered particles is found. In this region bubbles escaping from the surface as well as coalescence of bubbles forming larger particles occurs.

In some images pre ripples can be observed in a stroke of about $1 \mu \mathrm{m}$ width just outside the track with regular ripples.

Bubbles consist of frozen liquid containing gas, and have a perfect spherical shape (diameters $20-90 \mathrm{~nm}$ ) due to the internal gas pressure. Clustered particles are larger, the largest in figure 16 are about $250 \mathrm{~nm}$ in diameter. Due to the higher fluence the surface temperature becomes higher and one can imagine that the facets of the particles are crystallographic planes which are formed during cooling in the ns- $\mu$ s range.

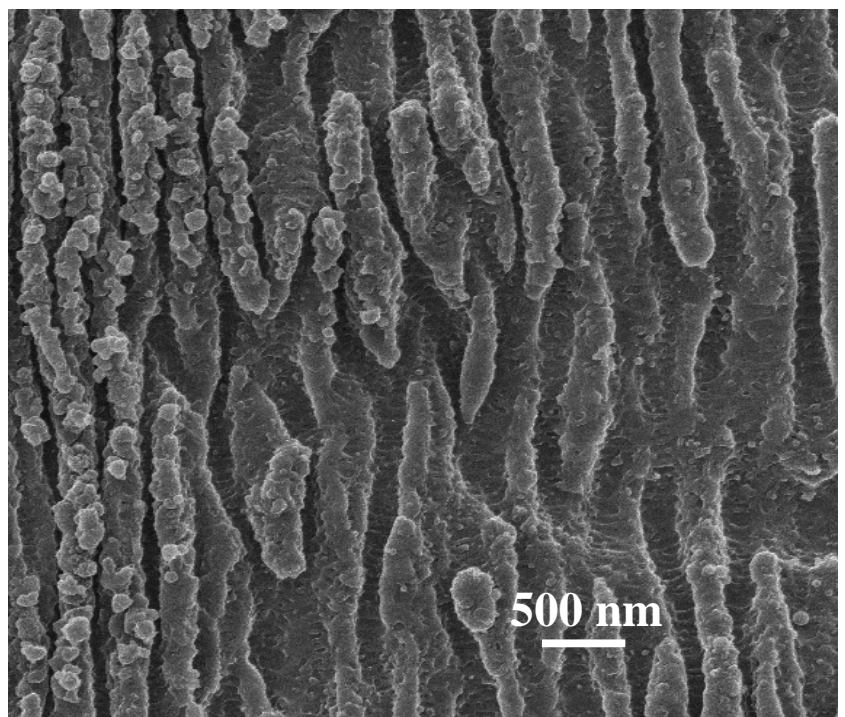

Fig.16 Edge of the laser track in fig.15. Coarse ripples with clustered particles. In the valleys of ripples the orientation of pre ripples is still visible.

It is observed that the valleys of regular ripples contain the remainders of pre ripples. Similar structures are observed for other materials: InP, $\mathrm{Si}, \mathrm{GaP}$ [9]. So probably also in many other materials pre ripples and the transition towards regular ripples occurs at low fluence levels.

\section{Sequence of events: bubbles, pre ripples and regular ripples}

The (solidified) mixture of gas and liquid will probably have a high refractive index and it can play a role as a local field concentrator and initiator of surface plasma waves. The interaction between bubbles and surface ridges as depicted in figure 6 , leads to pre ripples connected with bubbles, see figure 8 . In bubbles the gas to liquid ratio will be higher than in pre ripples. The images suggest that pre ripples have more viscous properties.

In this study we have clearly seen that at low average fluence levels starting at $13 \mathrm{~mJ} / \mathrm{cm}^{2}$ bubbles are formed, whereas a double scan generates pre ripples interacting with bubbles.

Bubbles trying to escape from a pre ripple have been observed frequently. After 10 scans with the same fluence or 10 overlapping pulses regular ripples start to appear, 
after 20 scans regular ripples and coalescence of bubbles become dominant. Off course the energy input in 10 scans is spread over a larger time span than a single scan with a 10 -times lower scan speed. The time between two pulses in a single scan with repetition rate $50 \mathrm{kHz}$ is $20 \mu$ s whereas the time between two scans is in the ms range. And besides this factor 1000 difference in time, there is also the uncertainty of relative pulse positions in different scans. This uncertainty can cause a certain, but in general small variation in fluence along a laser track.

Multiple scans give the same sequence of features as overlapping pulses. Starting with a single (low energy) pulse only bubbles are obtained. This is the condition in the upper right corner in figures 2, 3 and 13. Going to more overscans or lower scan speed produces first fine ripples in strong interaction with bubbles. Only after 20 overscans or a factor 16 speed reduction, regular ripples become the dominant surface feature.

A scheme of the different morphological phenomena after irradiation of silicium is given in [10] showing separated fluence regimes for bubbles and ripples. Similar phenomena are observed in this study with steel (alloy $800 \mathrm{H}$ ) as specimen material. The strong interaction of pre ripples with bubbles as depicted in figures $8-11$, has not been observed before.

\section{Dicussion}

The sequence of events with increasing fluence is bubbles that change towards a co-existence of bubbles with pre-ripples and finally regular ripples. A description of the electromagnetic interaction of a laser pulse with a metallic surface is required to start the process of understanding this sequence of events. Starting with a relatively flat surface bubbles are generated with a preference to align on surface ridges (figure 6) and we observe that the alignment of bubbles is a first sign of the orientation of pre ripples. When a second pulse arrives at the surface the bubbles are already solidified and obviously the interaction of subsequent laser pulses with aligned bubbles generates pre ripples. From the similarity in orientation and dimensions of bubbles on surface ridges and pre ripples we conclude that probably the same physical mechanism is active.

The nano sized laser induced periodically surface structures (LIPSS) are found after laser machining with the lowest fluence levels. Only by careful inspection of a surface the nano sized bubbles and pre ripples are found. Therefore we can imagine that in the past nano sized textures are missed. This idea is supported by careful inspection of figures with regular ripples elsewhere in literature e.g. [9]. So probably also in many other materials pre ripples and the transition towards regular ripples occur. Therefore lower fluence levels are an attractive process parameter to obtain self ordered nanostructures. Creation of a high degree of order with a large number of (very) low energy pulses is an attractive way to generate a higher degree of ordering. The potential of this approach is demonstrated in [11] where 40 $\mathrm{nm}$ wide grooves with an orientation perpendicular to the laser polarization direction are formed in synthetic singlecrystal diamond surfaces by machining below the ablation threshold.
With increasing fluence pre ripples transform into regular ripples. The physical mechanism for this transition is unclear; however one may argue that with higher fluence also thermal effects will become stronger. The importance of thermal effects is supported by the observation that the time interval between the first and second pulse is not critical. A time interval of microseconds and of milliseconds cause more or less the same effects, compare figures 8 and $14 \mathrm{~d}$.

The physics behind the orientation of pre ripples and the transition towards regular ripples need further attention starting with a thorough description of the interactions of a single pulse with electrons and phonons in a metal.

\section{Conclusions}

1. At the lowest fluence, $13 \mathrm{~mJ} / \mathrm{cm}^{2}$ on average, with an energy intensity of about $122 \mathrm{GW}$ per $\mathrm{cm}^{2}$, small bubbles with diameters $20-50 \mathrm{~nm}$, are formed preferentially on surface ridges.

2. With increasing fluence aligned bubbles connected to pre ripples, wavelength $122 \mathrm{~nm}$, with an orientation parallel to the polarization direction of the laser beam, transform into regular ripples with an orientation perpendicular to laser polarization.

3. Images of regular ripples in literature give reason to believe that pre ripples are formed in many materials at low fluence levels.

4. At the highest fluence $4.1 \mathrm{~J} / \mathrm{cm}^{2}$ clustered particles with diameters of about $250 \mathrm{~nm}$ are found presumably due to coalescence of bubbles.

\section{Acknowledgments}

(1) BSIK NanoNed funding for Scanning Helium Ion Microscopy is acknowledged.

(2) Support of TNO's KAVOT programme is acknowledged

(3) fs laser experiments by Lightmotif, Ronald Sipkema, are acknowledged.

\section{References}

[1] Bert Huis in 't Veld, Max Groenendijk, Hartmut Fischer, JLMN, 3, (2008) 206

[2] J.E.Sipe, J.F.Young, J.S.Preston, H.W. van Driel, Physical Review B, 27, (1983), 1141

[3] T.Aste, U.Valbusa, New Journal of Physics 7 (2005) 122

[4] R.Hill, J.Notte, B.Ward, Physics Procedia (2008)135141

[5] K. Sokolowski-Tinten, J. Bialkowski, A. Cavalleri, D. von der Linde, A. Oparin, J. Meyer-ter-Vehn, S.I. Anisimov, Physical Review Letters, 81, (1998) 224

[6] L.J. Lewis, D. Perez, Applied Surface Science, 2007.

[7] J.Reif, F.Costache, M.Bestehorn, Recent advances in Laser Processing of Materials, 275-290, Elsevier 2006

[8] Question raised by J.Reif at LAMP 2009, Kobe, Japan

[9] A.Borowiec, H.K.Augen, Applied Physics Letters, 82, (2003) 4462

[10] J.Bonse, S.Baudach, J.Krüger, W.Kautek, M.Lenzner, Applied Physics A 74, (2002), 19

[11] M.Shinoda, R.R.Guttass, E.Mazur, J.Applied Physics, 105, 053102 (2009)

(Received: October 7, 2009, Accepted: December 25, 2009) 